\title{
The Disconnect in Extended Forecast Graphic A Survey of Broadcast Meteorologists and their Public
}

\author{
Adapted From "Perception and \\ Comprehension of the Extended \\ Forecast: A Survey of Broadcast \\ Meteorologists and the Public," \\ by Jacob R. Reed (University of \\ Alabama) and Jason C. Senkbeil. \\ Published online in BAMS, February \\ 2020. For the full, citable article, see \\ DOI:10.1175/BAMS-D-19-0078.1.
}

$\mathrm{T}$ hough television is quickly being overtaken by smartphone weather apps as the primary source of weather information for the general public, it remains important for many, especially people 55 and older. However, nonproprietary research concerning the comprehension, clarity, and efficacy of TV weather graphics has been limited and warrants further study.

Previous research about these graphics has centered on public understanding of products such as tornado watches, the use of colors in radar displays, and tropical cyclone track and surge graphics. Less is known about viewer perception and understanding of weather forecast graphics on television news broadcasts. The primary graphic of concern in our research is the extended forecast graphic (EFG), which can extend out to 10 days. Our results reveal a disconnect between broadcast meteorologists and their audience.

\section{The important, but flawed, EFG}

Most EFGs across the country are formatted similarly, with vertical panels containing the day of the week, forecast high and low temperatures, weather icons, and a probability of precipitation (PoP). EFGs are highly sought after on television and online. This graphic is a cornerstone of local television weather broadcasts, often as a "hook" to 
increase viewership, and thus sponsorship. This paper lays the groundwork for understanding more about how local television weather forecasts are formatted, what graphics are shown, what information is shown on graphics, and the length of time devoted to each graphic.

PoPs often confuse viewers, but they are included in almost every weather forecast in all media. The confusion persists particularly during the warm season, when precipitation is often dominated by airmass convection. Not even meteorologists agree about what a PoP represents or how it should be used. The NWS defines PoP as a combination of the forecaster's confidence that precipitation will occur somewhere in the forecast area and the percent of the forecast area that will receive measurable precipitation. Assuming the forecaster's confidence level is $100 \%$, the PoP strictly expresses how much of the forecast area will receive measurable precipitation. Surprisingly, in one survey, more than $70 \%$ of meteorologists had a different interpretation of the PoP from this NWS definition. Broadcast meteorologists exhibited the lowest confidence in their interpretation of a PoP, yet studies show that broadcast meteorologists need to explain PoP in each forecast.

Studies have shown that though the public generally wants PoPs, they feel other parts of the precipitation forecast are more important. Thus, perhaps PoPs could be modified or removed to reduce confusion. Other variables, like timing, intensity, and duration of the precipitation were listed as more important, but these aspects of the forecast are rarely presented on the EFG. Thus, we set out to answer four questions about EFG:

1) What forecast variables does the public prioritize, and are they included on the EFG?

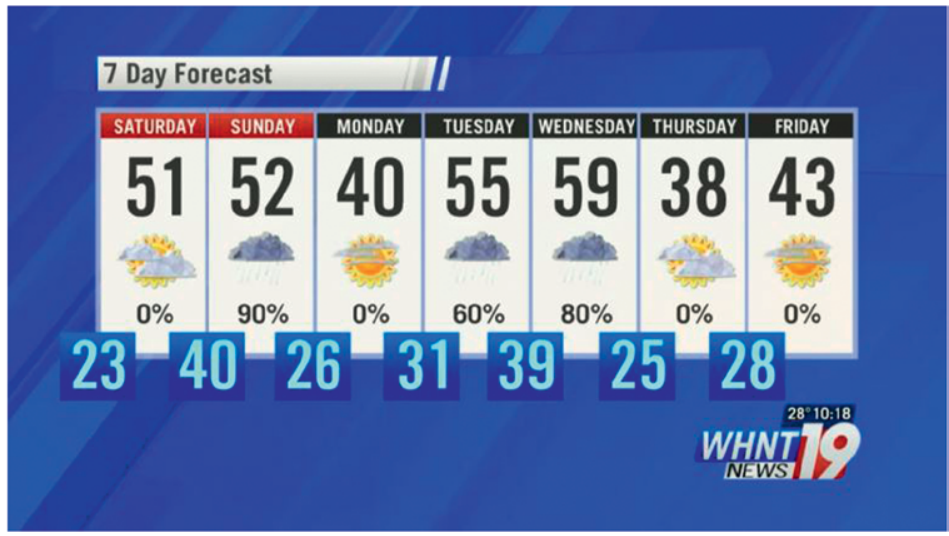

$\Delta$ * A 7-day extended forecast graphic shown during a local news broadcast.

\section{Chi-square test results for variables the public want to see in precipitation forecasts. Respons- es were grouped into low priority (ranks 1-3) and high priority (ranks 4-5). The disproportion- ality between the groups is statis- tically significant $(N=152) \cdot \frac{*}{\nabla}$}

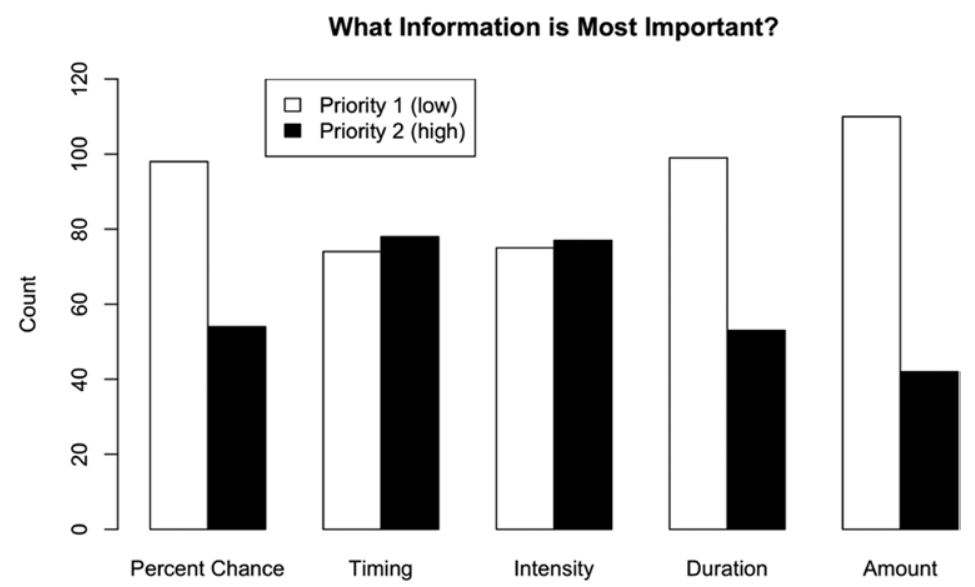

2) To what extent can the public comprehend and answer questions about a forecast after looking at an EFG?

3) What weather graphic do broadcasters say is most important to their audience and why?

4) For what length of time is the EFG shown during local weather broadcasts on television?

\section{Methods and data}

A 33-question survey consisting of free-response and Likert-scale questions, forecast scenarios, and EFG interpretations was created in Qualtrics. The survey was distributed by Qualtrics to a database of their users who were at least 30 years old (the demographic most likely to get weather information from television). The survey was limited to residents of Alabama, Georgia, Mississippi, or Tennessee, where precipitation occurs yearround. The survey was completed by 158 people.

Respondents for the public survey were evenly distributed in age groups 30-39, 40-49, 5059 , and $60+$. Females composed $77 \%$ of our sample, but women are more likely to watch local news. A total of $76 \%$ of those surveyed listed their ethnicity as White/Caucasian. Most participants reported themselves as having advanced education, with only $5 \%$ possessing less than a high school diploma. The sample represents a mixture of urban and suburban zip codes.

Approximately $77 \%$ of respondents say they check their local weather forecast at least once per day, looking at conditions most frequently on local television (28\%), followed closely by smartphone weather apps (21\%). A total of $86 \%$ of respondents reported that their source of weather information includes a PoP, and $91 \%$ say they pay attention to it.

Participants were shown EFGs and asked what they liked or disliked about each EFG. Survey participants also ranked the following 
precipitation forecast variables from most important to least important: timing, intensity, duration, amount, percent chance (PoP).

Previous research found that the public considered timing and intensity most important in precipitation forecasts. One question in our survey tested how robust those findings were, and to determine whether there was confusion around the forecast presentation and the ability of participants to correctly interpret the provided data, respondents were shown an EFG and asked questions about it.

In addition, a 13-question survey for broadcast meteorologists about the EFG, its use, and its effectiveness was developed using Qualtrics. We solicited participation twice through a private online group of more than 2,500 broadcast meteorologists, and the National Weather Association joined in the effort by emailing their database of members. Unlike the public survey, no geographic restrictions or age restrictions were implemented. Only 113 broadcast meteorologists completed the survey. Part of this study involved viewing features of EFGs used in 60 television weather segments from 20 television stations during the summer of 2018. Since the segment times did not follow a normal distribution, we tested for significant differences in the length of time the EFG was shown across each of the time categories.

Broadcast meteorologists from 35 states and the District of Columbia participated. The state with the highest number of respondents was Texas, with 12. Most broadcasters surveyed were between the ages of 25 and 34 (47\%) or 35 and $44(24 \%)$.

\section{The confusion of EFGs}

The results suggest that, in general, EFGs can be confusing and don't prioritize or contain information the public feels is most important. EFGs are not designed to communicate hazardous or otherwise atypical weather, and rarely, if ever, show nighttime weather. Timing was the precipitation factor most important to the public, followed by intensity.

After viewing a sample EFG in the survey, $72 \%$ of respondents were not sure what time it would rain on a specified day, $86 \%$ were not sure how much rain would fall on that day, and $79 \%$ were not sure how much rain would fall between two specified consecutive days. Most concerning was the inability of the clear majority of respondents to correctly discern the timing of expected

\section{- Sample negative criticisms of the EFG} from viewers surveyed:

\author{
"It does not give enough information." \\ "Some key points are missing." \\ "The low temperatures are a little confusing as to what day they apply." \\ "It is not easy to understand the numbers since they are not labeled in \\ any way." \\ "It does not tell if there will be storms, or if some of the rain will change \\ to snow."
}

\section{Sample positive comments about the EFG from broadcasters surveyed:}

\author{
"7-day forecast; It has the most information and everyone expects it in \\ every forecast." \\ "If I was told I could only show one graphic, that's the one I would choose." \\ "7-day forecast as that's the number one [reason] why people watch." \\ "This graphic's importance is underscored by the number of sponsors in \\ markets across the country."
}

precipitation, the forecast variable ranked as most important by the respondents. This was most likely the case because there was no information displayed in the provided EFG specifically including that information. Without the timing information, members of the public are left to guess and fill the information gaps on their own.

The results also show that the public interpretation of PoPs often differs from what broadcast meteorologists are intending to convey, and are consistent with that variable being among the most misunderstood by the general public. The public respondents almost always interpret each day's single weather forecast icon in the EFG differently from what the forecaster intended. For example, an icon with sunshine and a small thunderstorm may be interpreted by the public as an assurance, rather than a chance, of precipitation.

In open-ended questions, 61 respondents (39\%) criticized the deficiencies of the EFG, mainly around the precipitation forecast deficiencies, though specific aspects of temperature forecasts were also considered problematic. Together, the neutral and positive comments were roughly as numerous as-but more general than-the number of critical comments.

Conflict with broadcasters' opinions Just more than half (53\%) of the broadcasters 


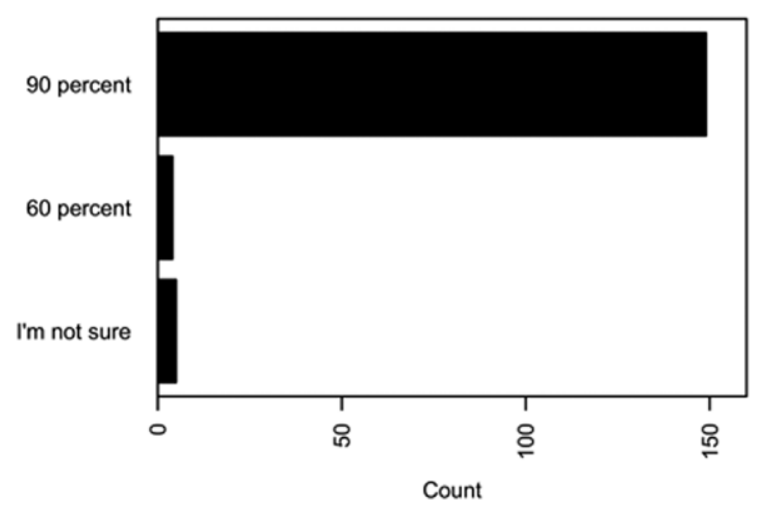

How much rain will fall Sunday?

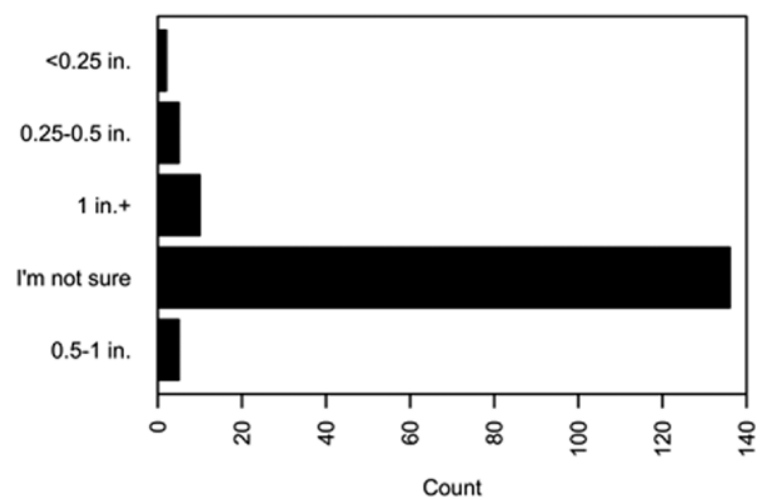

How much rain Tuesday-Wednesday?

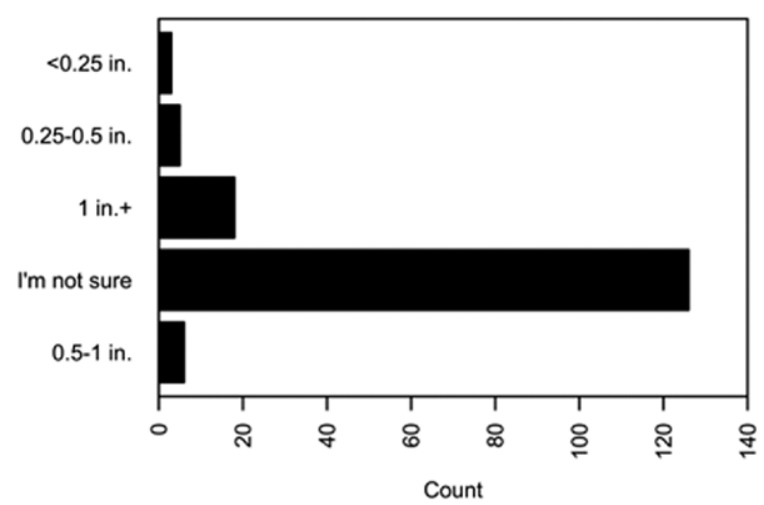

named the EFG as the most important graphic shown to their audiences; the short-term forecast was the next most frequently named graphic (13\%). Opinion on the PoP was is split: $47 \%$ did not believe the PoP is an effective way of communicating precipitation forecasts, $44 \%$ did. However, broadcasters overall praised the EFG, with many referring to it as "the number one reasons viewers watch.” This mainly

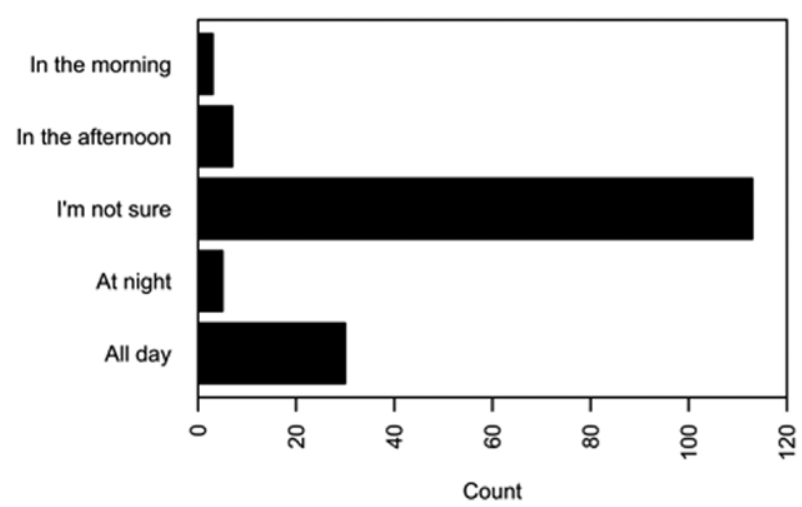

What will Sunday's low be?

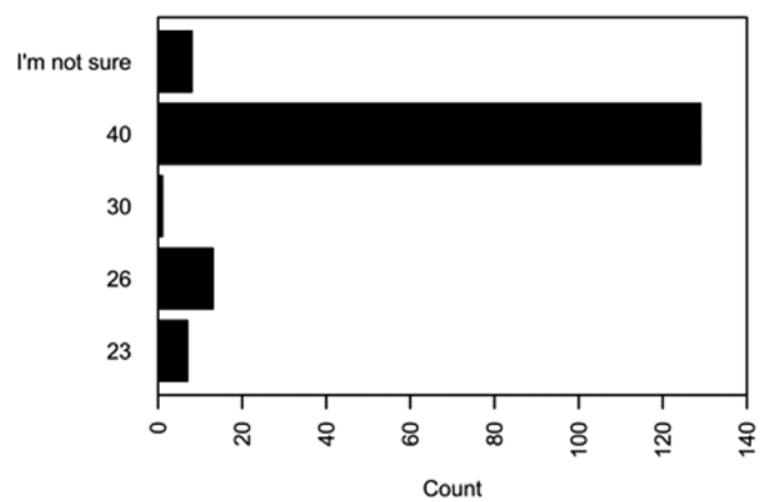

favorable perspective seems to directly conflict with the findings of the public survey.

Broadcasters put commendable effort into their EFG, and the specific forecast variables may be important in a given weather situation. The high level of detail presented earlier in the weather broadcast, however, is lost in translation in the EFG.

The design of the EFG may not be the only factor contributing to confusion. The length of time the EFG is shown during a weather broadcast and the order in which it is shown relative to other graphics (decisions very often not made by the broadcasters) are also important when evaluating the EFG. The evening broadcast EFGs we sampled showed the EFG between 10 and 40 seconds on screen (with a mean just under 20 seconds). But weekday morning broadcasts showed the graphic a mean of not quite 13 seconds, and the noon and midday broadcasts were less than a second more than that. In our survey of the public, respondents were given unlimited time to view the EFG, and still many people could not answer questions about it. 
BAMS: What would you like readers to learn from this article?

Jacob Reed: A theme emanating from meteorology conferences and workshops has been consistency in messaging across the weather enterprise. Message consistency reduces confusion among members of the public. The extended forecast graphic, probability of precipitation, and other graphics are commonly misunderstood, so it is important to begin these discussions to make progress. Particularly, broadcast meteorologists and weather websites where the EFG is displayed need to think about their graphics and how the message might be interpreted and possibly misinterpreted.

BAMS: How did you become interested in the topic of this article?

JR: I had been working as a broadcast meteorologist for five years when I began to start thinking more critically about the graphics I was showing each day-did they make sense? Was what I was hoping to convey actually getting across? Where I saw what I believed to be the biggest disconnect between me and my audience was during the summertime when afternoon thunderstorms were almost a guarantee in the U.S. Southeast. It was clear to me that the percentage chance of precipitation, or PoP, was a big source of confusion. In most cases, the use of PoPs is most frequent on the extended forecast graphic.

BAMS: The use of PoPs has long been debated, yet your survey shows the communications context matters.

\section{JR: Initially, I wanted to limit my scope to just this forecast variable, however I ended up taking a broad- er look at the graphic as a whole. This led to the discovery that not only did the PoP cause confusion, but many other elements on the graphic itself did too.}

BAMS: What surprises/surprised you the most about the results of this study?

\section{JR: I had suspected that certain} elements of the extended forecast graphic might be a source of confusion or easily misinterpreted. Percentages, icons, and even words can mean very different things to different people-and the public's perception of the forecast might vary greatly from what the meteorologist intended. What surprised me most, however, was that even the forecast temperatures present on this graphic-the low temperature, in particular - could create confusion. Those who took the sur- vey had some trouble locating the correct forecast low temperature for a given day. As someone who prepared this graphic hundreds of times, that was an element that I never gave a second thought about whether it was confusing or not.

BAMS: What was the biggest challenge you encountered while doing this work?

JR: One goal of this paper was to begin amassing peer-reviewed research to help all broadcasters create more effective graphics and improve communication. We anticipate the real challenges will appear if some members of the meteorological community begin to change their products based on these suggestions but other members refuse. New products must be vetted and tested and also supported by all members of the meteorological enterprise.

BAMS: What's next? How will you follow up?

JR: A related manuscript tests four new versions of the extended forecast graphic with the intent of minimizing confusion. These new extended forecast graphics were based on feedback from the first survey from broadcast meteorologists and the public.
The EFG is sometimes shown near the end of the newscast, separately from the main weathercast time. This is usually dependent on the time remaining at the end of the newscast, which can be highly inconsistent. If the EFG is shown at the end of the newscast, it is typically for less time than during the main weather broadcast, which was analyzed in this research. On rare occasions, if a broadcast meteorologist is asked to fill time at the end of the newscast, the EFG might be shown for at least 30 seconds or more. In these instances, however, the meteorologist is typically not on camera, and this may introduce other limitations to effective communication.
There are still many questions left to answer-for example, about the design of the EFG. Our findings can be used to improve the graphical communication by including the information people want. Factors that can make weather icons a source of confusion are similar to what makes the PoP incomprehensible: Does the icon represent the most inclement weather expected? Is it an average? Can a single icon represent $24 \mathrm{~h}$ of weather?

If it is so important, its efficacy must improve. In a separate work, we will test the efficacy of modifications to the EFG. 
AMS titles now

available as eBooks

at springer.com

\section{AMS BOOKS}

\section{RESEARCH APPLICATIONS HISTORY}

www.ametsoc.org/amsbookstore

Springer

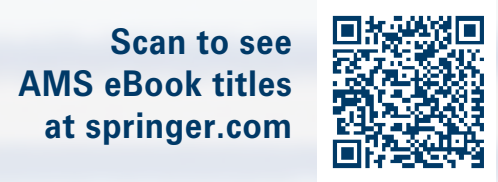

Springer

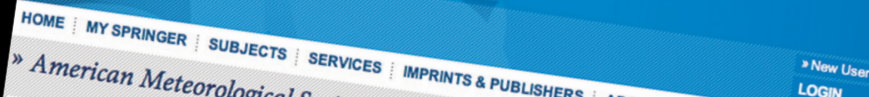

"American Meteorological SOCiety
"AOMTIN

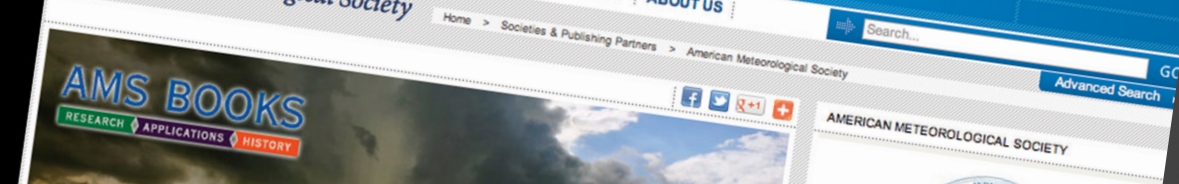

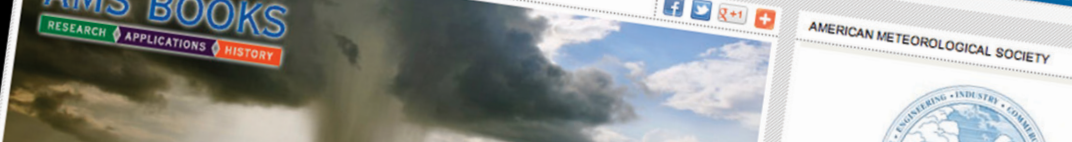

American Meteorol

Promoting the dewerogical Society

the atmos the deverap

the atmospheric and relont and disseminatb of

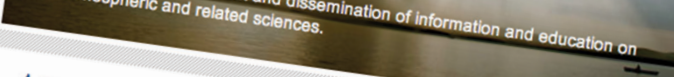

American Meteorological Society eB

AMS Books

published by the AMs a monograph publishing prent

shing program related to ho poer-

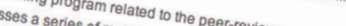

related to the as eBooks) as weil ar ariy out-ortprint vol metoorological and historica journals

to the atmospheric sciences a growing list of academis, which have been urough ographs

ind policy, and general-interest books

Sort listing by:

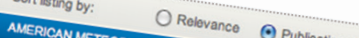

वध्ये Exporit to $\mathrm{CSO}$

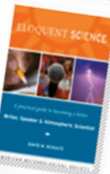

Eloquent Science

Bloquent Scie

A Practical Guite

Schulta, David
2009

Price from $\$ 45.00$

copyright Year

Avalobe

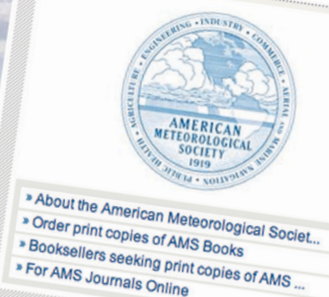

RESOURCES

Stay Informed

Receive notitication of now releases from
Books

Sub

AMS 